\section{Contrastive Analysis of Pre-and Post-functioning of the Yueqing City Tuberculosis Control Project \\ $\mathrm{Xu}$ Boyou \\ Yueqing Hygiene and Disease-Preventing Station, Zhejiang, PEOPLE'S REPUBLIC OF CHINA}

Introduction: Tuberculosis is a serious and infectious disease harmful to human health. Since 1990, tuberculosis has occurred frequently, and has not been controlled effectively. Tuberculosis is one of important infectious diseases in our state's "nine-five" infectious disease control plan, so fulfilling the task of preventing and curing tuberculosis is our obligation.

Methods: For controlling tuberculosis effectively, we implemented a united management strategy for this disease, enhancing the ratio of exact diagnosis and cure, thus fulfilling the purpose of this project.

Results: Tuberculosis may be controlled effectively by: (1) enhancing the understanding, responsibility, and the importance of preventing this disease, (2) improve the propagation and citizen's self-protection; and (3) manage uniformly patients with tuberculosis; (4) carry out methods of WTO cure generally, and (5) develop investigation of tuberculosis and enhancing the ratio of exact diagnosis, etc. Key words: analysis; control; infectious diseases; plan; treatment; tuberculosis

Prehosp Disast Med 2001;16(2):s86.

\section{Retinal Detachment after Intraocular Foreign Body Removal with Vitrectomy Yan Hua; Xu Yinghai; He Tiangeng; Zhang Jingkai; Yao Baoqun}

Tianjing Medical University, Tianjing, PEOPLE'S REPUBLIC OF CHINA

Objective: To report the incidence of retinal detachment after intraocular foreign body removal with vitrectomy, and to analyze the relative reasons.

Methods: A retrospective analysis of 20 consecutive patients (20 eyes) who underwent pars plana vitrectomy for the removal of intraocular foreign body was conducted .A standardized, three port vitrectomy was used in all injured eyes. Results: Four (20\%) of 20 eyes had retinal detachment. Visual acuity increased in 14 eyes (70\%), stabilized in 3 eyes $(15 \%)$, and deteriorated in 3 eyes $(15 \%)$.

Conclusion: The possible reasons of retinal detachment after intraocular foreign bodies removal with vitrectomy included: (1) the remnant vitreous traction formation, (2) not proper photocoagulation around the retinal hole, (3) tiny retinal hole not found during the surgery, (4) no preoperative photocoagulation on the surface of retina around the foreign body, and (5) the peripheral retinal tear induced by removal of a large foreign body.

Key words: detachment; foreign bodies; intraocular; removal; retina; vitrectomy

Prehosp Disast Med 2001;16(2):s86.

\section{Investigation on Lens Condition of $370 \mathrm{Han}$ Nationality Officials Living at an Altitude 4,000 M Yang Jie \\ Guoluo People's Hospital, Qinghai, PEOPLE'S REPUBLIC OF CHINA}

Cataract disease is a worldwide ophthalmological disease and is correlated to living conditions, living habits, natural environment, and geographical location, but not to nation, nationality, race, or sex. Patients who suffer from cataract disease almost always live in the highlands. Deliquescence of this disease is very long; it most often occurs in adults and the elderly.

In China, the number of persons who are blind as a result of cataract disease is about 4 million. Control of cataract disease is one of three rehabilitation projects, according to the disease control plan of the Ministry of Health of China. Through our investigation of 337 persons, we conclude that there are three reasons for lens turbidity: (1) strong radiation exposure to infrared and ultraviolet rays., (2) metabolization turbulence and lens nutrition decompensation, and (3) incretion function turbulence and a genetic factor .

Key words: blindness; causes; cataract; lens; turbidity Prehosp Disast Med 2001;16(2):s86.

\section{Investigating Injuries among Middle and Primary School Students in Huaiyuan County Ye Dongqing; Huang Fen; Chen Wenhua; Hao Jiabu; Zhang Xiujun; Sun Benmei}

Department of Epidemiology, Anhui Medical University, Hefei, PEOPLE'S REPUBLIC OF CHINA

Objective: To investigate the level of injuries and the epidemiological features of injuries among middle and primary students, in order to find out the effective intervention measures for preventing injuries. Methods: A questionnaire was used to investigate the injuries sustained by 15,149 middle and primary school students.

Results: There were 12 kinds of injuries, the total rate of injuries was $39.0 \%$. The top three kinds of injuries included: injuries from falls (15.9\%), bitten by animals $(15.2 \%)$, and knife-cutting or sharp weapon wounds $(11.5 \%)$. The rate of injuries among junior middle school students was the highest (51.7\%), that of high school students was lower (45.2\%), and that for primary school was the lowest. The rate of injuries was related closely to the kind of school. Conclusion: Injury has affected the studies and health of middle and primary school students. We must strengthen supervision, safety education, and increase awareness on self-protection among the students.

Key words: bites; children; cuts; epidemiology; falls; injuries; prevention; schools; students; types

Prehosp Disast Med 2001;16(2):s86. 